\title{
LA DIVISIÓN DEL TRABAJO SOCIAL DE DURKHEIM*
}

\author{
Robert K. Merton
}

\begin{abstract}
RESUMEN
La fuente de la vida social es, según Durkheim, la similitud de las consciencias y la división del trabajo. La primera es más evidente entre las sociedades primitivas, en las que la «solidaridad mecánica», puesta de manifiesto por la ley represiva, prevalece. La segunda es propia de las sociedades avanzadas, en las que se manifiesta una mayor "densidad dinámica» y en las que las reglas jurídicas definen la naturaleza y las relaciones de las funciones. Al combatir el individualismo y basar la existencia de las sociedades sobre el "consenso de las partes», Durkheim refuta su énfasis positivista que niega la relevancia de los fines en el estudio científico de la sociedad. En su discusión de los fines sociales hay una tendencia antimecanicista latente. La teoría del desarrollo unilineal está basada sobre datos etnográficos deficientes. Asume la ausencia de división del trabajo entre las sociedades primitivas, así como la de la «solidaridad mecánica» entre las sociedades modernas. Las leyes represivas y restitutivas se usan como índices de la solidaridad mecánica y orgánica, pero Durkheim no establece con ninguna precisión las perfectas relaciones que él da por sentadas entre sus tipos de solidaridad y de leyes.
\end{abstract}

En un estilo pedestre, y de alguna manera impropio, la obra de Durkheim De la division du travail social ha merecido una tardía traducción al inglés, cuarenta años después de su publicación inicial ${ }^{1}$. Este testimonio de la continua

* Publicado originalmente en American Journal of Sociology, vol. 40, n. 3 (nov. 1934): 319-328.

1 George Simpson, Emile Durkheim on the Division of Labor in Society, New York: Macmillan Co., 1933. Las citas siguientes se refieren a esta edición. 
estima otorgada a la obra de Durkheim provee un incentivo para reconsiderar la primera magnum opus de este protagonista hegemónico de la escuela sociológica. El valor de este examen es doble: por una parte, permite una reconsideración del papel desempeñado por Durkheim en el desarrollo del pensamiento sociológico moderno y, por otra, llama la atención acerca de varias concepciones fundamentales para gran parte de la investigación contemporánea.

A la hora de valorar sus contribuciones, es pertinente un análisis del contexto teórico en el que esta obra fue escrita. Firmemente vinculado a la corriente del pensamiento positivista que procede de Comte, el libro de Durkheim expresa muchos de sus rasgos característicos. Así, busca adoptar los métodos y criterios de las ciencias físicas para la determinación de aquellas leyes sociales inducidas mecánicamente, las cuales, bajo condiciones dadas, se obtienen con una ineludible necesidad. Explícito en este proceder está, desde luego, la asunción de la viabilidad del mismo, así como la susceptibilidad del fenómeno social a ser objeto de tal estudio. De este modo, se pasa por alto el hecho de que el concepto de causalidad, tal vez más marcadamente en las ciencias sociales que en las físicas, es una asunción epistemológica, una cuestión de imputación y no de observación. Dentro de esta tradición positivista, La división es clasificable, además, como un ejemplo del enfoque antiindividualista y antiintelectual. Es una rebelión declarada contra el positivismo utilitarista e individualista que, buscando sus prototipos en los sistemas de Hobbes y Locke, caracterizó a gran parte del pensamiento social inglés. Un sociologismo radical le pareció a Durkheim una forma de mantener la autonomía de la sociología como una disciplina independiente, y a esta preocupación dominante se deben muchas de sus concepciones. De especial trascendencia es el hecho de que La división, aunque bosqueja muchas de las ideas que Durkheim desarrollará con posterioridad ${ }^{2}$, muestra un enfoque objetivo, con reservas implícitas, del cual él divergió claramente más tarde, especialmente en sus Formes élémentaires de la vie religieuse.

Los periplos de las ideas expresadas en La división han llegado a este país, pero un breve sumario es, sin embargo, conveniente para establecer las bases de la discusión. Sostiene Durkheim que la fuente de la vida social es doble: la similitud de las consciencias y la división del trabajo. En un tipo de sociedad que él llama "primitiva», la solidaridad es ocasionada por una comunidad de representaciones que dan lugar a leyes que imponen a los individuos creencias y prácticas uniformes bajo la amenaza de medidas represivas. Estas leyes represivas son índices externos - esto es, observables en un sentido positivista- de la "solidaridad mecánica». Por otro lado, la división del trabajo social, que si bien intensifica no impone la individualización, también ocasiona una «solidaridad orgánica», basada en la interdependencia de los individuos y los grupos que actúan cooperativamente. Este tipo de solidaridad es indiciada por reglas jurídicas que definen la naturaleza y las relaciones de las funciones. Estas reglas

El punto de partida de El suicidio es explícito en el libro II, capítulo I, de La división...; Las reglas del método sociológico, en las pp. 349 y ss.; y Las formas elementales..., en las pp. 288 y ss. 
podrían ser adecuadamente denominadas como leyes restitutivas, dado que su violación no implica consecuencias expiatorias, sino sólo reparadoras. Históricamente, el movimiento ha sido desde la solidaridad mecánica a la orgánica, aunque la primera nunca desaparece completamente. La causa determinante de esta tendencia se encuentra en el incremento del tamaño y densidad de las poblaciones con el habitual - si no invariable - y concomitante incremento de la interacción social. De este modo se intensifica la lucha por la existencia, puesto que sólo por medio de la diferenciación progresiva de funciones es posible la supervivencia para muchos que, de otra manera, estarían condenados a la extinción. Esta continua tendencia acontece mecánicamente a través de unas series de equilibrios dinámicos sociales que se interrumpen y se restablecen.

Ahora bien, como se sugirió previamente, Durkheim busca combatir el positivismo individualista que ignora la relevancia de los fines sociales como determinantes parciales de la acción social. De ahí que se viera enfrentado a un dilema perturbador: como positivista, para admitir la irrelevancia de los fines para el estudio de la sociedad; como antiindividualista, indicar la eficacia de los propósitos sociales en el condicionamiento de la acción social y, con ello, abandonar el positivismo radical. Porque si, como el positivismo nos ha hecho creer, la lógica y la ciencia pueden tratar sólo de hechos empíricos, entonces una ciencia de los fenómenos sociales edificada sólo sobre esta base se convierte en imposible, dado que esta actitud relega al limbo todos los fines, a saber, las anticipaciones subjetivas de los sucesos futuros, sin cuya consideración la conducta humana se vuelve inexplicable ${ }^{3}$. Fines, metas y propósitos no son, por definición, datos lógicoexperimentales, sino, más bien, juicios de valor; y, sin embargo, una comprensión de los fenómenos sociales requiere un estudio de su rôle $e^{4}$. Esto no implica un compromiso teleológico-determinista, sino que simplemente apunta al hecho de que los fines subjetivamente concebidos — con independencia de su reconocimiento de todos los datos pertinentes en una situación dada-, así como las "condiciones externas», influyen en la conducta. Excluir los fines por considerarlos «impropios» para el estudio científico no es eximir a la sociología de metafísica, sino contaminar sus hallazgos con una metafísica tosca y acrítica ${ }^{5}$.

3 Paradójicamente, esta postura es admitida por el positivista V. Pareto. Ver su Traité de sociologie générale, II, París, 1917, pp. 1349 y ss. También, Talcott Parsons, «Some Reflections on "The Nature and Significance of Economics" ", Quarterly Journal of Economics, XLVIII (1934), 511-545. Tengo una profunda deuda con el Dr. Parsons por mucho de lo aquí expuesto.

${ }^{4}$ Véase Heinrich Rickert, Kulturwissenschaft und Naturwissenschaft, Tübingen: Hohr, 1921, pp. 99 y ss. Léon Duguit, cuyas concepciones de la similitud a través de la división del trabajo recuerdan estrechamente a las de Durkheim, presenta una exposición brillante de la trascendencia de los fines para la interpretación. Véase su libro L'État, le droit objectif et la loi positive, Fontemoing, 1901, pp. 33 y ss. En nuestro país, la exposición más exacta de esta posición se encuentra en W. I. Thomas y F. Znaniecki, The Polish Peasant in Europe and America (University of Chicago Press, 1918-1920), especialmente la discusión de las actitudes sociales y «la definición de la situación».

${ }^{5}$ Véase C. Hartshorne y P. Weiss (eds.), Collected Papers of Charles Sanders Pierce, I, Harvard University Press, 1931, pp. 52 y ss. 
En el momento de escribir La división, Durkheim era demasiado positivista como para reconocer explícitamente toda la fuerza de esta posición, pero, no obstante sus doctrinas metodológicas expresas, subrepticiamente se desliza entre los dos brazos del dilema y salva su consciencia antiindividualista al ocuparse de los fines sociales. Así pues, indica de manera clara que si la sociedad fuera simplemente una resultante de individuos yuxtapuestos llamados a relaciones contractuales provisionales para la satisfacción de sus respectivos intereses inmediatos, esto es, si la relación social típica fuera la económica, entonces no tendríamos por más tiempo una sociedad, sino el «estado de la naturaleza» de Hobbes.

«Allí donde el interés es la única regla, dado que nada refrena los egoísmos presentes, cada individuo se encuentra en pie de guerra frente a sus semejantes y cualquier tregua no sería de larga duración» ${ }^{6}$.

Esto corresponde a la descripción de Durkheim de la anomia. Pero el hecho es, continúa el autor, que incluso en sociedades tan altamente contractuales e individualizadas como las nuestras, este estado bruto de naturaleza no prevalece. Entonces, ¿qué es lo que evita esta situación que, como cabría esperar, caracterizaría a una sociedad contractual si el enfoque individualista fuera válido? Es el "consenso de las partes», la integración de los fines individuales, el complejo-valor social ${ }^{7}$. Esto se ve claramente en la regulación legal de los contratos entre individuos, ya que si bien es verdad que estos contratos son inicialmente un asunto voluntario, una vez iniciados, están sujetos a la sociedad como una "tercera parte» controladora y omnipresente. A través de un sistema de derecho, un órgano de control social, el acuerdo de las voluntades individuales se constriñen en consonancia con las funciones sociales difusas. Además, en este proceso la sociedad desempeña un papel activo para determinar qué obligaciones son "justas», a saber, las que están de acuerdo con los valores sociales dominantes, y cuáles no necesitan su puesta en vigor. Con este incisivo análisis, Durkheim refuta una de las doctrinas básicas de una sociología atomística, puesto que encuentra en la propia relación que había sido considerada individualista por excelencia la interpenetración significativa de los factores sociales ${ }^{8}$.

Su concepción es similar a la tensión hacia la consistencia y la autonomía de las mores de Sumner, así como a la noción de Goldenweiser del límite a las discrepancias entre los diversos aspectos de una cultura. Esta concepción de la sociedad va vinculada a un reconocimiento del papel previamente mencionado

${ }^{6}$ La división..., pp. 203-204; cf. p. 365.

7 Ibid., p. 360. Cf. Parsons, op. cit., p. 517.

${ }^{8}$ La distinción entre el análisis de Durkheim y las teorías del contrato social debería estar, de este modo, totalmente clara. Como el propio Durkheim observó: «Sólo una crítica singularmente superficial podría reprochar a nuestra concepción de la coerción la reedición de las teorías de Hobbes y Maquiavelo». Las reglas..., p. 151. 
de los fines sociales y a la aceptación de la doctrina de la emergencia. Que la conducta social no puede explicarse mediante la referencia a los comportamientos individuales como mera yuxtaposición es mantenido tanto por Durkheim como por Pareto 9 , y es precisamente este criterio el que se esgrime para justificar el carácter de la sociología como disciplina distintiva.

En la discusión de Durkheim de los fines sociales está latente una tendencia antimecanicista. Cuando los instrumentos son forjados con miras al intento de consecución de los fines, a causa de este mismo hecho las condiciones se desarrollan de tal forma que no sólo actúan en la dirección de las metas, sino que reaccionan ante éstas y, frecuentemente, cambian las orientaciones de los valores. Estas nuevas valoraciones pueden exonerar al hombre de la necesidad de aceptar las «condiciones de la existencia» —el milieu de Durkheim- y de actuar de una manera previamente determinada. Habiendo cambiado su «definición de la situación», su conducta tiene una orientación nueva, y el determinismo mecanicista, basado en el conocimiento de los factores objetivos, no da ya razón apropiada de esta conducta. Pero, como suele ser característico de los teóricos mecanicistas, Durkheim no distingue adecuadamente sus concepciones abstractas, en este caso las condiciones externas de la existencia, de la situación concreta, en la que se incluye a los factores normalmente suprimidos de la selección humana de objetivos. Él piensa que las ineludibles conclusiones que se derivan de su delineación abstracta de la situación representan hechos reales en toda su diversidad empírica ${ }^{10}$. Para expresarlo de otra manera, Durkheim evita deliberadamente tratar sus concepciones como construcciones ideales que exijan una modificación apropiada antes de que puedan describir adecuadamente fenómenos sociales concretos.

En su presentación de la evolución social, Durkheim afirma rastrear genéticamente la transición desde la solidaridad mecánica a la orgánica, y aquí es cuando sus defectuosos datos etnográficos le llevan a equivocarse. Como Maine y Steinmetz, le gusta observar el predominio, incluso la existencia

9 Es especialmente chocante que Pareto, con su inclinación hacia el empirismo, adoptara esta opinión. Tratado..., I, p. 26: «Notez qu'etudier les individus ne veut pas dire que l'on doit considérer plusieurs de ceux-ci mis ensembles, comme une simple somme; ils forment un composé, lequel, á l'égal des composés chimiques, peut avoir des propriétés qui ne sont pas la somme des propriétés des composants». Esta concepción es, por supuesto, señalada en todos los escritos de Durkheim, si bien su primera exposición apareció en Las reglas..., p. 126. (Nota del traductor: Si bien para respetar el sentido original del texto he optado por dejar la frase de Pareto en francés, tal y como Merton la tomó, la consideración al lector en castellano y la relevancia de la cita me lleva a traducirla a continuación: «Reparen en que estudiar a los individuos no quiere decir que se deban considerar como una mera agregación de muchos de ellos, como una simple suma; ellos forman un compuesto, el cual, al igual que los compuestos químicos, puede tener algunas propiedades que no son la suma de las propiedades de los componentes».)

${ }^{10}$ Hace tiempo que Hume había percibido esta confusión de la ciencia mecanicista. El profesor A. N. Whitehead indica este error con la descriptiva frase de «la falacia de la concreción equivocada». Véase su libro Science and the Modern World, New York: Macmillan, 1931, pp. 75 y ss. Una aguda descripción psicológica de la base de este error se encuentra en el libro de Richard Avenarius, Kritik der reinen Erfahrung, II, Leipzig: Reisland, 1907-8, pp. 376 y ss. 
exclusiva, de la ley penal en la sociedad primitiva. En realidad, como recientes estudios de campo han demostrado, las sociedades primitivas también poseen un corpus de leyes civiles restitutivas, impuestas mediante mecanismos sociales, que involucran derechos y deberes entre individuos ${ }^{11}$. La existencia de estas relaciones esencialmente contractuales entre los pueblos primitivos desvirtúa la plausibilidad de la teoría de Durkheim del desarrollo unilineal. Además, al afirmar el predominio de la solidaridad orgánica en las sociedades modernas, Durkheim tiende a despreciar, indebidamente, el persistente factor de la comunidad de intereses. Este sesgo pervierte su análisis de los elementos de cohesión social. Factores de integración de grupo tales como las concepciones del honor - Ehre- y la subsunción del individuo bajo intereses colectivos durante periodos de guerra y conflicto - elementos importantes en la cohesión de las sociedades contemporáneas ${ }^{12}$ — , son generalmente ignorados de manera injustificada por Durkheim en su empeño por encontrar en la división del trabajo la única fuente de la solidaridad moderna. La inviolada unidad del grupo se convierte en indispensable durante los conflictos intersociales, y esta unidad se consigue en gran parte mediante las apelaciones a los sentimientos comunes. Asimismo, la noción no jurídica del honor es un poderoso, por no decir siempre efectivo, mecanismo regulador que fomenta la cohesión social. El hecho de que estas formas de solidaridad mecánica subsistan todavía sugiere razones adicionales para rechazar el argumento de Durkheim del desarrollo unilineal.

La concepción de Durkheim de esta evolución unilineal debe, por otra parte, reconsiderarse a la luz de lo que ha sido convenientemente llamado el "principio de los límites» del desarrollo ${ }^{13}$. El desarrollo en una dirección dada podría continuar hasta llegar a ser contraproducente, después de lo cual ocurriría una reacción en la dirección opuesta. Si Durkheim no hubiera intentado extrapolar más allá del universo de sus datos, podría haber encontrado un exponente de esta reacción en los siempre más frecuentes estados de anomia que acompañan al incremento de la división del trabajo. En el mundo económico, es preciso observar los movimientos de refortalecimiento que se dan después de alcanzarse grados óptimos de diferenciación para comprender que el proceso no es necesariamente unidireccional.

Para llegar a esta concepción de la evolución, Durkheim no necesita, como se ha afirmado, abandonar su posición sociológica. Es verdad que él encuentra la "causa determinante» del incremento de la división del trabajo en el crecimiento e intensificación de la densidad poblacional, que es principalmente un

${ }_{11}$ Bronislaw Malinowski, Crime and Custom in Savage Society, New York: Harcourt, Brace \& Co., 1926, pp. 55 y ss. Contrástese con la afirmación de Durkheim de que «en las sociedades primitivas... la ley es enteramente penal». La división..., p. 76.

12 Véase Georg Simmel, Soziologie, München y Leipzig: Ducker \& Humblot, 1923, pp. 202, 404 y ss.

${ }_{13}$ Véase A. A. Goldenweiser, «History, Psychology and Culture», Journal of Philosophy, Psychology and Scientific Methods, XV (1918), 593; P. A. Sorokin, "The Principle of Limits», Publications American Sociological Society (1932), pp. 19-28. 
factor biológico, pero el cambio especificado sólo tendrá lugar en la medida en que este cambio demográfico esté vinculado a una mayor interacción social y a la concomitante intensificación de la competencia. Es, pues, este factor social —la «densidad dinámica», como él lo denomina- el que Durkheim encuentra verdaderamente determinante. En un escrito posterior, reafirma este punto de forma aún más categórica al advertir que la densidad poblacional y la densidad dinámica no siempre van asociadas - por ejemplo, en China-y que en estos casos el incremento de la división del trabajo se ve considerablemente inhibido $^{14}$. Por lo tanto, debe revisarse la fórmula superficial que sólo atribuye una diferenciación creciente de funciones a los cambios demográficos. En la medida en que esta diferenciación es generalizable como un proceso social, podría decirse que está asociada con la competición entre individuos y entre grupos, con independencia de los factores que conduzcan a tal competición.

Si abandonamos la teoría unilineal de Durkheim nos quedamos con una aguda caracterización de dos sociedades, mecánica y orgánica, tomadas como tipos ideales o como ficciones heurísticas. Así pues, éstas podrían considerarse como casos límite, nunca prevalecientes en la realidad empírica, que podrían ser empleados de manera feraz como polos de referencia hacia los que orientar teóricamente los datos empíricos. De este modo, la obra de Durkheim provee un esquema conceptual que podría ser ventajoso en la interpretación de los procesos de diferenciación, integración, competición y otros de similar estilo.

Otro aspecto de la metodología de Durkheim, que caracteriza no sólo a La división sino también a sus obras posteriores, es el uso de «índices», que él considera como la traducción "externa», mensurable, de los hechos sociales «internos», no directamente observables. De la misma manera que el físico mide el calor y la electricidad a través de ciertos fenómenos objetivamente observables y fácilmente mensurables, tales como la subida y la bajada del mercurio en un tubo de cristal y la oscilación de la aguja de un galvanómetro, así Durkheim espera emplear la ley represiva y restitutiva como índices, respectivamente, de la solidaridad mecánica y orgánica ${ }^{15}$.

En este punto surge una dificultad fundamental. Si los hechos observados $(L)$ han de ser índices significativos y relativamente precisos de los tipos de solidaridad $(S)$, las relaciones siguientes deben ser verdaderas. Sea $L(x, y \ldots)$ una función de cantidades mensurables $(x, y \ldots)$ (estadísticas de leyes penales o restitutivas) asociada a $S\left(x^{\prime}, y^{\prime} \ldots\right)$ (el hecho social-la cohesión social) que satisfacen estos postulados: cuando $L$ varía de una manera determinada, $S$ varía de forma equivalente. Cuando hay incrementos sucesivos en $L$, el primer cambio

${ }^{14}$ Las reglas..., p. 140: «Hemos cometido el error, en nuestra División del trabajo, de subrayar en exceso la afirmación de que la densidad material es expresión exacta de la densidad dinámica». Paul Barth yerra de manera manifiesta al adscribir a Durkheim a una interpretación materialista de la historia sin modificar. El cambio de Durkheim al idealismo se hace más evidente en su obra sobre la religión. Véase P. Barth, Die Philosophie der Geschichte als Soziologie, Leipzig: Reisland, 1922, pp. 628-642.

15 La division..., p. 66. 
de $L$ es de $L_{1}$ a $L_{2}$ y el segundo de $L_{2}$ a $L_{3}$, de tal modo que el primer incremento es mayor que el segundo y, por tanto, también el primer incremento en $S$ (la solidaridad) es mayor que el segundo. Este postulado debe mantener su validez aun cuando sustituyamos «mayor» por «menor» ${ }^{16}$. Esto permite una variación concomitante entre los hechos sociales y sus índices, siendo las variaciones de los primeros inmensurables y relativas a las variaciones directamente mensurables de los últimos.

Durkheim fracasa precisamente en la demostración de este tipo de relaciones puesto que no establece con precisión aquellas asociaciones perfectas que asume van a darse entre sus tipos de solidaridad y de leyes. Por ejemplo, la solidaridad orgánica podría regularse por los usos consuetudinarios y las costumbres sin llegar nunca a convertirse en ley civil. Éste fue el caso especialmente de una buena parte de la Edad Media ${ }^{17}$. Además, como se ha sugerido, mucha de la solidaridad mecánica en la sociedad contemporánea — que se manifiesta, por ejemplo, en el «honor»- no encuentra expresión en la ley represiva. Estas indicaciones, necesariamente breves, son suficientes para destacar las discutibles premisas sobre las que Durkheim basa su sistema de índices ${ }^{18}$.

En su capítulo sobre la división del trabajo y la felicidad, en general brillante, Durkheim evidencia otra debilidad fundamental de su método. Elimina ciertas explicaciones posibles de un conjunto particular de fenómenos sociales al demostrar que las consecuencias lógicas de las teorías rechazadas no concuerdan con los hechos observados. Da por hecho que el número posible de teorías explicativas es determinable, $x$, y que habiendo eliminado $x-1$ explicaciones obtiene la solución necesariamente válida. De este modo, Durkheim sostiene que "el deseo de llegar a ser más feliz es la única fuente individual que puede dar cuenta del progreso (de la división del trabajo). Si aquélla se desestima, no queda ninguna otra ${ }^{19}$. Este método del experimento proyectado fue destacado por Descartes, con quien Durkheim estaba manifiestamente en deuda. Descartes mantuvo que en la aproximación a la realidad nos encontraremos con que muchas consecuencias resultan de los principios inicialmente asumidos y que la consideración racional decidirá cuáles de estas consecuencias se realizan ${ }^{20}$. Pero la falacia de este método radica en la asunción inicial de que alguien puede agotar la totalidad de las explicaciones posibles. La eliminación de las teorías alternativas de ningún modo incrementa las probabilidades de las restantes.

16 Véase A. L. Bowley, The Mathematical Groundwork of Economics, Oxford: Clarendon Press, 1924, pp. I y ss.

${ }_{17}$ Véase Paul Vinogradoff, "Customary Law», en The Legacy of de Middle Ages, G. C. Crump y E. F. Jacob (eds.), Oxford: Clarendon Press, 1927, pp. 287-319.

${ }_{18}$ El mismo tipo de crítica debe dirigirse contra los índices de cohesión del grupo y de desintegración que emplea Durkheim en El suicidio.

${ }_{19}$ La división..., p. 251. Está claro que esto es una afirmación extrema, pero para Duguit, op. cit., pp. 50 y ss., sugiere una explicación individualista y no eudemónica.

${ }^{20}$ René Descartes, «Discours de la méthode», Oeuvres, VI, París, 1902, pp. 64 y ss. 
De La división del trabajo social, de Durkheim, puede en general decirse que presenta un análisis sugerente e incisivo de un determinado proceso social y de sus correlatos estructurales. Si sus conclusiones son demasiado radicales, si su método es a veces defectuoso, todavía podría reconocerse, desde la posición ventajosa que proporcionan cuatro décadas de ulterior investigación, que el libro permanece como una de las contribuciones cimeras de la sociología moderna.

(Traducido por Cristóbal Torres Albero.) 


\section{DEBATE}

